\title{
Relational mobility predicts social behaviors in 39 countries and is tied to historical farming and threat
}

\author{
Robert Thomson a, Masaki Yuki ${ }^{b, 1}$, Thomas Talhelmc, Joanna Schug ${ }^{d}$, Mie Kito ${ }^{\mathrm{e}}$, Arin H. Ayanian ${ }^{\mathrm{f}}$, Julia C. Becker ${ }^{\mathrm{g}}$, \\ Maja Becker ${ }^{h}$, Chi-yue Chiu', Hoon-Seok Choi', Carolina M. Ferreirak, Marta Fülöp ${ }^{1, m}$, Pelin Gul ${ }^{\mathrm{n}}$, \\ Ana Maria Houghton-lllera ${ }^{\circ}$, Mihkel Joasoo ${ }^{p}$, Jonathan Jong ${ }^{q, r}$, Christopher M. Kavanagh $^{q}$, Dmytro Khutkyy ${ }^{\mathrm{s}}$, \\ Claudia Manzi ${ }^{\mathrm{t}}$, Urszula M. Marcinkowska ${ }^{\mathrm{u}}$, Taciano L. Milfont ${ }^{\mathrm{v}}$, Félix Neto ${ }^{\mathrm{w}}$, Timo von Oertzen ${ }^{\mathrm{x}}$, Ruthie Pliskin ${ }^{\mathrm{y}}$, \\ Alvaro San Martin ${ }^{2}$, Purnima Singh ${ }^{\text {aa }}$, and Mariko L. Visserman ${ }^{\text {bb }}$
}

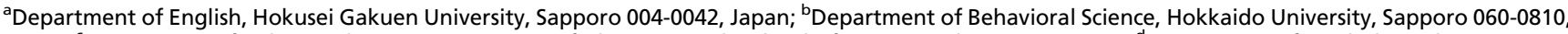
Japan; 'Department of Behavioral Science, University of Chicago Booth School of Business, Chicago, IL 60637; 'Department of Psychological Sciences, College of William \& Mary, Williamsburg, VA 23185; ${ }^{e}$ Department of Sociology and Social Work, Meiji Gakuin University, Tokyo $108-8636$, Japan; ${ }^{f}$ Institute for Interdisciplinary Research on Conflict and Violence, Bielefeld University, Bielefeld 33615, Germany; ${ }^{9}$ Institute of Psychology, Universität Osnabrück, Osnabrück 49074, Germany; ${ }^{h}$ CLLE, Université de Toulouse, CNRS, UT2J, Toulouse 31058, France; 'Department of Psychology, Chinese University of Hong Kong, Shatin, NT, Hong Kong SAR, The People's Republic of China; 'Department of Psychology, Sungkyunkwan University, Seoul 3063, Republic of Korea; ${ }^{k}$ Developmental and Educational Psychology, University of Castilla - La Mancha, Albacete, Albacete 2002, Spain; 'Institute of Cognitive Neuroscience and Psychology, Hungarian Academy of Sciences, Budapest 1117, Hungary; mInstitute of Psychology, Eötvös Loránd University, Budapest H-1053, Hungary; ${ }^{\mathrm{n}}$ School of Psychology, University of Kent, Canterbury, Kent CT2 7NP, United Kingdom; ${ }^{\circ}$ Colegio Colombiano de Psicólogos, Bogotá 110221 , Colombia; ${ }^{P}$ Department of Psychology, University of Tartu, Tartu 50090, Estonia; IInstitute of Cognitive and Evolutionary Anthropology, University of Oxford, Oxford OX2 6PE, United Kingdom; ' Centre for Advances in Behavioural Science, Coventry University, Coventry CV1 5FB, United Kingdom; ${ }^{5}$ Electronic Democracy

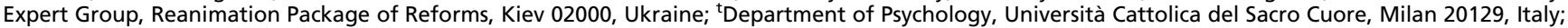
unstitute of Public Health, Collegium Medicum of the Jagiellonian University, Kraków 31-531, Poland; ${ }^{v}$ Centre for Applied Cross-Cultural Research, Victoria University of Wellington, Wellington 6140, New Zealand; " ${ }^{w}$ Department of Psychology, University of Porto, Porto 4200-135, Portugal; ${ }^{x}$ Institut für Psychologie, Universität der Bundeswehr, Munich 85579, Germany; ${ }^{y}$ Institute of Psychology, Leiden University, Leiden 2333 AK, The Netherlands; ${ }^{z}$ Managing People in Organizations Department, IESE Business School, Madrid 28010, Spain; aaDepartment of Humanities and Social Sciences, Indian Institute of Technology Delhi, Delhi 110016, India; and ${ }^{\mathrm{bb}}$ Department of Experimental and Applied Psychology, Vrije Universiteit Amsterdam, Amsterdam 1081 BT, The Netherlands

Edited by Susan T. Fiske, Princeton University, Princeton, NJ, and approved May 31, 2018 (received for review August 3, 2017)

\begin{abstract}
Biologists and social scientists have long tried to understand why some societies have more fluid and open interpersonal relationships and how those differences influence culture. This study measures relational mobility, a socioecological variable quantifying voluntary (high relational mobility) vs. fixed (low relational mobility) interpersonal relationships. We measure relational mobility in 39 societies and test whether it predicts social behavior. People in societies with higher relational mobility report more proactive interpersonal behaviors (e.g., self-disclosure and social support) and psychological tendencies that help them build and retain relationships (e.g., general trust, intimacy, self-esteem). Finally, we explore ecological factors that could explain relational mobility differences across societies. Relational mobility was lower in societies that practiced settled, interdependent subsistence styles, such as rice farming, and in societies that had stronger ecological and historical threats.
\end{abstract}

relational mobility | culture | socioecology | multicountry | interpersonal relationships

n some societies, relationships are mostly fixed. People have stable and long-lasting relationships, but they have little choice when it comes to friends, family, and romantic partners. Other societies work more like free agent markets. Relationship options are abundant, meaning that people can freely seek out new partners and leave old friends behind. For decades, sociologists (1), economists (2), psychologists (3), and anthropologists (4) have tried to understand why societies have different relationship "markets" and how these differences set the ground rules for cooperation, social exchange, and norms. Behavioral ecologists have found that fluid social markets have more partner choice, which increases cooperation in humans (5) and even birds and insects (6).

Within this backdrop, we introduced the concept of relational mobility to quantify variance in partner choice in human societies (reviewed in refs. 7 and 8). Relational mobility is a socioecological variable (9) that represents how much freedom and opportunity a society affords individuals to choose and dispose of interpersonal relationships based on personal preference $(7,10)$. Societies with low relational mobility have less flexible interpersonal relationships and networks; people form relationships based on circumstance rather than active choice. In these societies, relationships are more stable and guaranteed, but there are fewer opportunities to find new relationships or leave unsatisfying ones $(11,12)$

In contrast, societies with high relational mobility give people choice and freedom to select and dispose of interpersonal relationships, which are based on mutual contract and are less guaranteed (12). High relational mobility societies are akin to open, choice-laden biological markets $(5,6)$, where people select partners based on selfinterest (13). A few early studies have found that relational

\section{Significance}

Biologists and social scientists have long tried to understand why some societies have more fluid and open interpersonal relationships-differences in relational mobility-and how those differences influence individual behaviors. We measure relational mobility in 39 societies and find that relationships are more stable and hard to form in east Asia, North Africa, and the Middle East, while they are more fluid in the West and Latin America. Results show that relationally mobile cultures tend to have higher interpersonal trust and intimacy. Exploring potential causes, we find greater environmental threats (like disease and warfare) and sedentary farming are associated with lower relational mobility. Our society-level index of relational mobility for 39 societies is a resource for future studies.

Author contributions: R.T., M.Y., M.K., A.H.A., J.C.B., M.B., C.-y.C., H.-S.C., C.M.F., M.F. P.G., A.M.H.-I., M.J., J.J., C.M.K., D.K., C.M., U.M.M., T.L.M., F.N., R.P., A.S.M., P.S., and M.L.V. designed research; R.T., M.Y., J.S., M.K., A.H.A., J.C.B., M.B., C.-y.C., H.-S.C., C.M.F., M.F., P.G., A.M.H.-I., M.J., J.J., C.M.K., D.K., C.M., U.M.M., T.L.M., F.N., R.P., A.S.M., P.S., and M.L.V. performed research; R.T. contributed new reagents/analytic tools; R.T., M.Y., T.T., and T.v.O. analyzed data; and R.T., M.Y., T.T., J.S., C.M.K., and T.L.M. wrote the paper. The authors declare no conflict of interest.

This article is a PNAS Direct Submission.

This open access article is distributed under Creative Commons Attribution-NonCommercialNoDerivatives License 4.0 (CC BY-NC-ND).

${ }^{1}$ To whom correspondence should be addressed. Email: myuki@let.hokudai.ac.jp.

This article contains supporting information online at www.pnas.org/lookup/suppl/doi:10. 1073/pnas.1713191115/-/DCSupplemental.

Published online June 29, 2018. 
mobility is high in North America and low in Japan and Hong Kong in east Asia as well as Ghana in west Africa $(7,14,15)$.

In a sense, relational mobility sets the "rules of the game" for social relationships. When a society sets a particular level of relational mobility, it makes certain behaviors and psychological tendencies more or less adaptive. Indeed, studies have found that differences in relational mobility can explain societal differences, such as generalized trust, self-enhancement, self-disclosure, intimacy, and need for uniqueness (7). In this way, previous studies have shown that relational mobility drives differences between societies in how people act, think, and feel $(8,16)$.

Despite a recent surge in interest in relational mobility, there are two important questions that researchers have yet to address. First, no work has explored antecedents of relational mobility - that is, why it is higher in some societies and lower in others. Second, a majority of previous studies exploring outcomes of relational mobility have been dual country, generally between Japan/Hong Kong and the United States/Canada. This raises the question of whether the processes identified in previous dual-country studies exist beyond the oft-documented and potentially idiosyncratic East-West dichotomy.

This study tests these questions. First, we measure relational mobility in 39 societies around the world. Second, we explore antecedents - the factors that might cause societies to be higher or lower in relational mobility. Third, we perform a number of confirmatory tests on consequences of relational mobility that previous studies have found. These analyses test the idea that relational mobility encourages certain behaviors and psychological tendencies across a wide range of disparate countries and regions. Fourth, we test the entire theory in a multilevel model of relational mobility that outlines links between distal environmental and relational structures at the societal level and proximal human behaviors and psychology at the individual level (Fig. 1).

\section{Results}

Cross-Cultural Validity of Measures. One concern in cross-cultural studies is whether we can measure constructs accurately across cultures and languages. Data from scales used in our multicountry survey evidenced reliability, measurement equivalence, and validity across societies (SI Appendix, section 1.2). All scales showed partial scalar invariance, indicating that participants in different countries responded to survey items in similar ways and that we can meaningfully compare scale averages across societies. The relational mobility scale (ref. 10 and SI Appendix, Table S1) showed high within-nation agreement $\left[M_{\mathrm{rwg}(\mathrm{j})}=0.92, \mathrm{SD}=0.02\right.$, Min $=0.87$, high variability between different societies [ICC $(1)=$ $0.09]$, and highly reliable society-level means [ICC $(2)=0.98]$ (Table $1)$. In short, these results suggest that people within each society tended to agree about how mobile their society is and that societies differ meaningfully in how relationally mobile they are.

Relational Mobility's Convergence and Antecedents. Below, we use publically available data and our own in-survey data to run a battery of exploratory tests examining the convergent validity and antecedents of relational mobility. For all exploratory analyses, we provide bootstrapped (5,000 samples) bias-corrected confidence intervals $(\mathrm{BCaCI}) . P$ values are also given to identify potential research goals for follow-up studies but do not indicate confirmatory hypothesis testing results.

Society-level socioecological variables

Individual-level variables

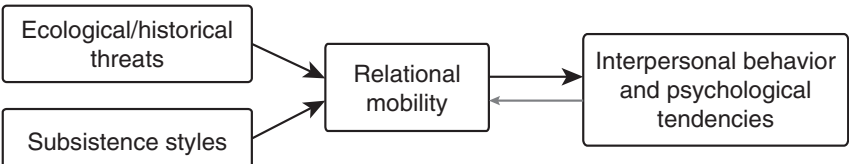

Fig. 1. Overall multilevel systems view of relational mobility.
Convergent and concurrent validity of the relational mobility measure. The relational mobility scale taps into respondents' perceptions of the opportunity and choice that people have in their interpersonal relationships in their society. Are people's perceptions accurate? We found that the societal-level relational mobility scores were correlated with other variables that reflect opportunity and freedom for relationships in societies. Relational mobility was associated with such variables as the justifiability of divorce $[r=0.51$ $(95 \% \mathrm{BCaCI}=0.18,0.79), P=0.007]$, the belief that marriage is outdated $[r=0.46(95 \% \mathrm{BCaCI}=0.11,0.72), P=0.033]$, attempts to poach romantic partners for long- or short-term relationships for women as well as men ( $r$ values $\geq 0.55, P$ values $\leq 0.098)$, lower importance placed on job security $[r=-0.58(95 \% \mathrm{BCaCI}=-0.24$, $-0.86), P=0.029]$, and residential mobility $[r=0.53(95 \% \mathrm{BCaCI}=$ 0.02, 0.83), $P=0.036$ ] (SI Appendix, Table S6). Furthermore, in multilevel analyses, relational mobility accounted for $18 \%$ of societal variance in how many romantic partners respondents had in the past as measured in our survey $(\beta=0.394, P=0.028)$ (SI Appendix, Table S7). These results suggest that perceptions of relational mobility do reflect the reality of interpersonal relationships in different societies, providing convergent validity evidence for the relational mobility scale (further discussion is in SI Appendix, section 1.2.5).

We then examined how relational mobility was associated with cultural variables that measure loose, independent vs. tight, interdependent cultures measured in previous studies. We found that relational mobility was correlated with loose cultural norms $[r=$ $0.65(95 \% \mathrm{BCaCI}=0.47,0.83), P=0.001]$, openness to multiple religious viewpoints [religious syncretism; $r=0.50(95 \% \mathrm{BCaCI}=$ $0.21,0.77), P=0.009]$, independent self-construal $[r=0.76(95 \%$ $\mathrm{BCaCI}=0.07,0.99), P=0.050]$, less hierarchy $[r=-0.46(95 \%$ $\mathrm{BCaCI}=-0.73,-0.13), P=0.041]$, valuing competition and personal improvement [performance orientation; $r=0.42(95 \%$ $\mathrm{BCaCI}=0.18,0.65), P=0.029]$, and less fate control $[r=-0.51$ (95\% BCaCI $=-0.73,-0.23), P=0.02]$ (SI Appendix, Table S8). Relational mobility was also correlated with sociopolitical variables, such as democracy $[r=0.42(95 \% \mathrm{BCaCI}=0.13,0.68), P=0.009]$, political rights $[r=0.34(95 \% \mathrm{BCaCI}=0.02,0.64), P=0.043]$, and civil liberties $[r=0.44(95 \% \mathrm{BCaCI}=0.15,0.70), P=0.008](S I$ Appendix, Table S6). The fact that relational mobility is correlated with these concepts gives evidence of concurrent validity for the relational mobility scale as a measure of the opportunity and freedom of relational choice within a society. These correlations suggest that places with higher relational mobility tend to have cultures that emphasize individual autonomy toward relationships and group memberships.

The distribution of relational mobility around the world. Overall, relational mobility was high in North America and low in East Asia, which replicates previous findings. We also found that Western Europe had high relational mobility as did Latin America, whereas the Middle East, North Africa, and South Asia had lower relational mobility. Relational mobility was highest in Mexico and Puerto Rico and lowest in Japan and Malaysia (Table 1 and Fig. 2).

Antecedents of relational mobility. Next, we explored factors that could cause differences in relational mobility. We first theorized that relational mobility would be lower in societies with more interdependent subsistence styles. On the one hand, farming cultures tend to be more sedentary and interdependent, with stable communities and labor exchange that put people in tight relationships with reciprocal duties (17). Among different crops, paddy rice is particularly interdependent, requiring tight coordination of labor and irrigation (18).

On the opposite side of the spectrum is herding. Herders move frequently, meaning that they have fewer stable, long-term relationships and more opportunities to form and break relationships. Studies have shown that herding cultures are more individualistic than nearby farming cultures (19). We found that societies that have historically devoted more crop land to paddy rice had lower relational mobility $[r=-0.48(95 \%$ $\mathrm{BCaCI}=-0.70,-0.17), P=0.003]$ and that societies with more herding land had higher relational mobility $[r=0.52(95 \%$ $\mathrm{BCaCI}=0.29,0.71), P=0.001]$. When we combined subsistence 
Table 1. Sample characteristics of the 39 countries and regions

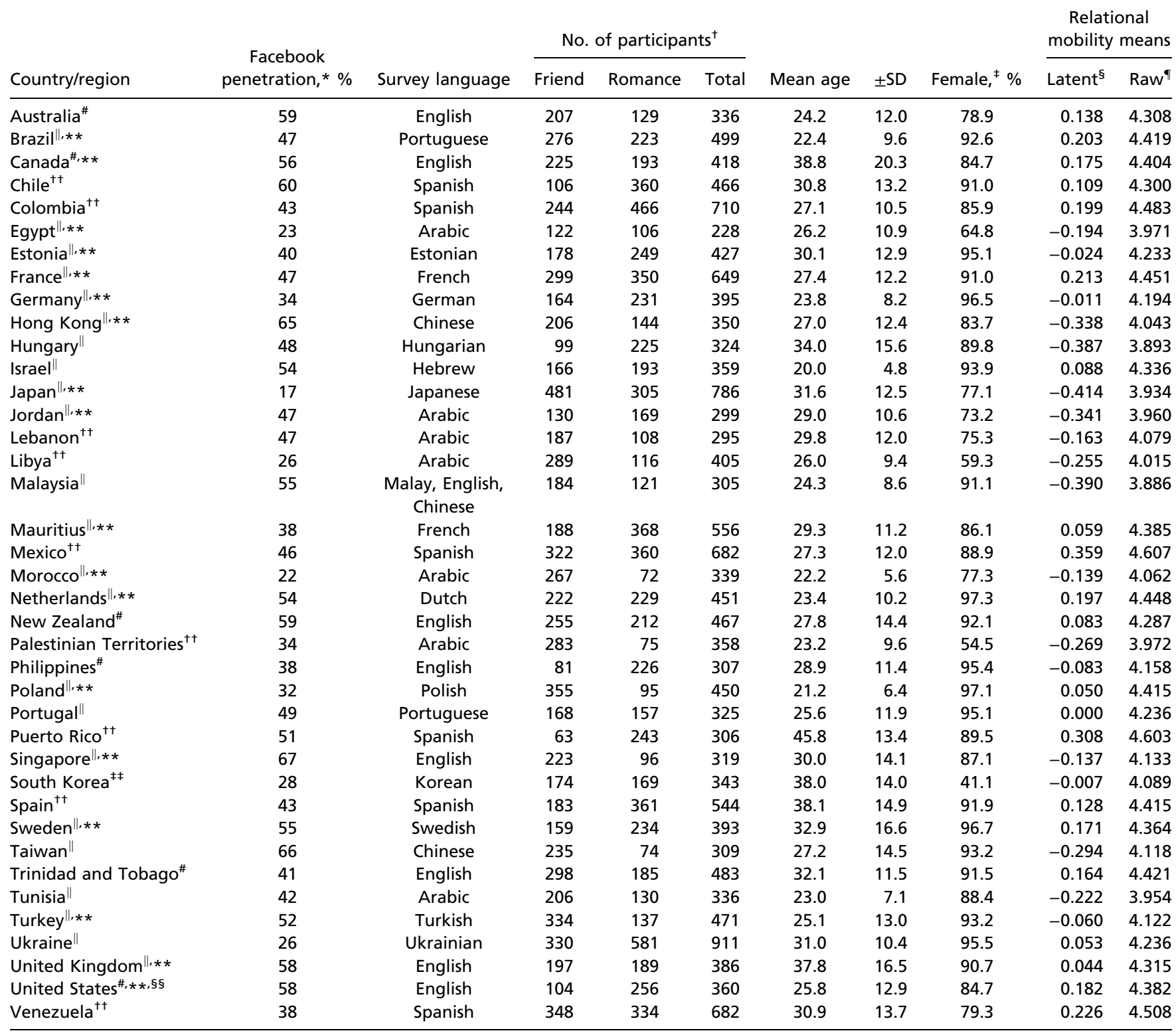

*As of January 2015 (35).

${ }^{\dagger}$ Friend refers to where the target of dependent variables was respondents' best friend; romance is where the target was a romantic partner (SI Appendix, sections 1.1 and 1.2).

${ }^{\ddagger} S I$ Appendix, section 1.1.1 has a discussion on gender.

§Portugal sample $=0.000$, response style adjusted (SI Appendix, section 1.2.3).

"Raw means are provided for reference only.

\#Data collection November 10-17, 2014.

"Data collection February 10-18, 2015.

**Data collection May 19-27, 2015.

${ }^{\dagger \dagger}$ Data collection July 26 to August 2, 2015.

${ }^{\ddagger \ddagger}$ Data collection June 3-12, 2016.

§§Data collection October 3-10, 2014.

styles into a single index (SI Appendix, section 1.5.3), results confirmed that cultures that practiced more interdependent subsistence styles have lower relational mobility $[r=-0.63(95 \% \mathrm{BCaCI}=-0.80$, -0.40), $P<0.001$ ] (Fig. 3 and SI Appendix, Table S10).

Second, we theorized that relational mobility should be lower in societies that have acute historical and ecological threats. Research suggests that a basic human response to threat is group cohesion and cooperation $(20,21)$, strong norms (22), and insularity (23). In short, regions that have faced more threats tend to have limited community sizes and less openness to outsiders. Thus, we expected that relational mobility would be lower in societies with high ecological threat.

Results showed that, indeed, relational mobility was lower in regions with critical environment and health vulnerabilities, including geoclimate harshness $[r=-0.45(95 \% \mathrm{BCaCI}=-0.63$, $-0.23), P=0.018]$, historical prevalence of pathogens $[r=-0.28$ $(95 \% \mathrm{BCaCI}=-0.55,-0.02), P=0.090]$, lives lost to tuberculosis $[r=-0.38(95 \% \mathrm{BCaCI}=-0.59,-0.15), P=0.019]$, and population 


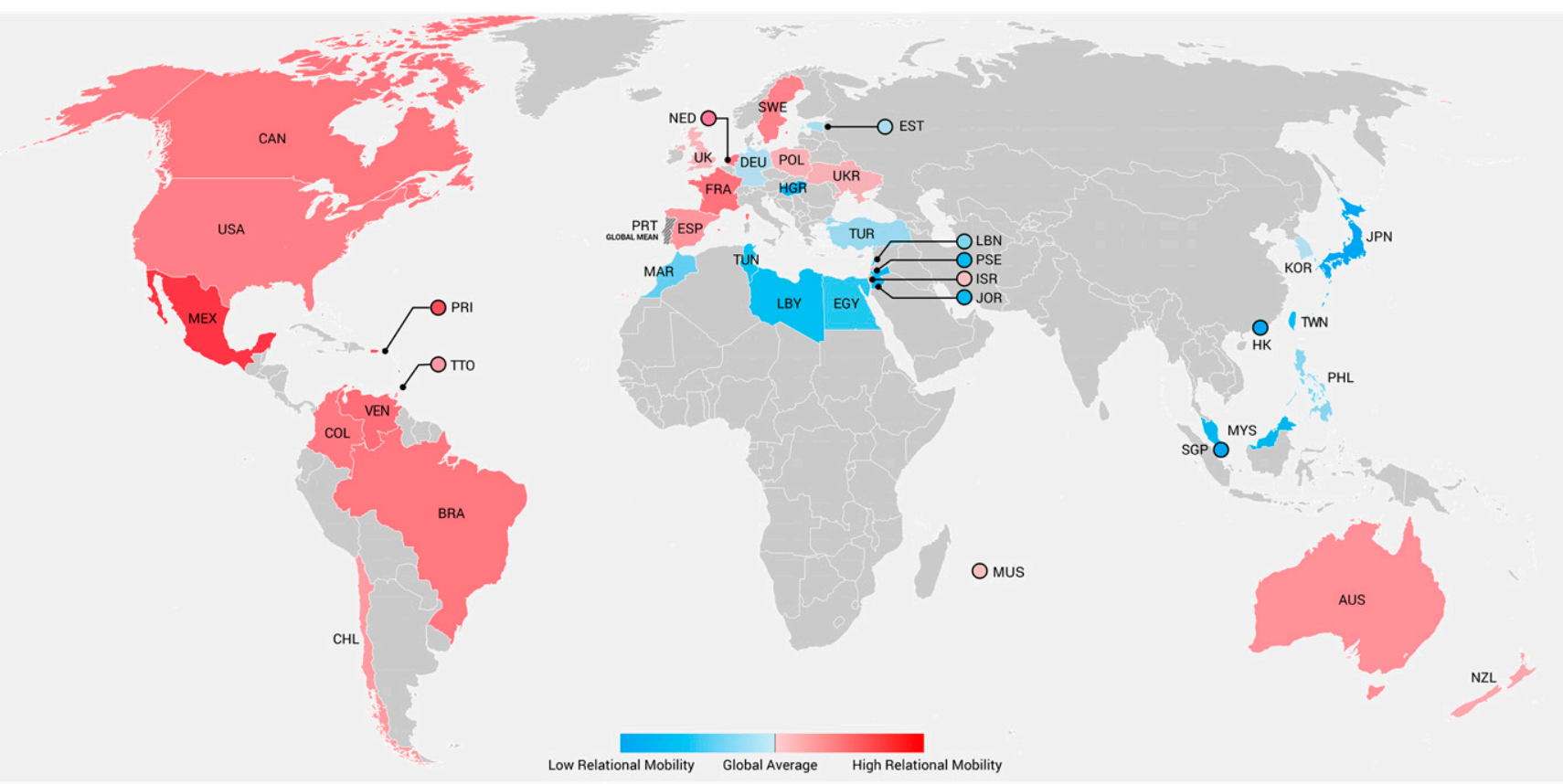

Fig. 2. Relational mobility society-level latent means in visual format. Blue indicates societies lower in relational mobility than the midpoint (Portugal). Red indicates societies higher in relational mobility than the midpoint. Fully interactive visualizations available online at relationalmobility.org. AUS, Australia; BRA, Brazil; CAN, Canada; CHL, Chile; COL, Colombia; EGY, Egypt; EST, Estonia; FRA, France; DEU, Germany; HK, Hong Kong; HGR, Hungary; ISR, Israel; JPN, Japan; JOR, Jordan; LBN, Lebanon; LBY, Libya; MYS, Malaysia; MUS, Mauritius; MEX, Mexico; MAR, Morocco; NED, The Netherlands; NZL, New Zealand; PSE, Palestinian Territories; PHL, Philippines; POL, Poland; PRT, Portugal; PRI, Puerto Rico; SGP, Singapore; KOR, South Korea; ESP, Spain; SWE, Sweden; TWN, Taiwan; TTO, Trinidad and Tobago; TUN, Tunisia; TUR, Turkey; UKR, Ukraine; UK, United Kingdom; USA, United States; VEN, Venezuela.

pressure, including population density both in $\mathrm{AD} 1500[r=-0.39$ $(95 \% \mathrm{BCaCI}=-0.62,-0.11), P=0.047]$ and in the present $[r=-0.39$ $(95 \% \mathrm{BCaCI}=-0.60,-0.14), P=0.029]$. Countries that were poorer historically (lower gross domestic product per capita in 1950) were less relationally mobile $[r=0.51(95 \% \mathrm{BCaCI}=0.33,0.69)$, $P=0.002]$ (SI Appendix, Table S10). We combined a number of these historical and ecological threats to form a single index (SI Appendix, section 1.4), and this predicted relational mobility well, even when taking into account current gross domestic product per capita $[r=-0.54(95 \% \mathrm{BCaCI}=-0.70,-0.38), P<0.001]$ (Fig. 4 and historical and ecological threat in SI Appendix, Table S10).

Relational Mobility's Consequences. Based on previous dual-country studies, we tested a number of confirmatory hypotheses about the psychological and behavioral outcomes of relational mobility in the 39 -society dataset. We theorize that generalized trust $(10,24)$ and self-esteem (25) should be higher in relationally mobile societies, because they give people confidence to approach new desirable people in an open and competitive interpersonal marketplace $(3,7$, $8)$. Hence, trust and self-esteem help people achieve the task of acquiring desirable relationships (16). Another consequence is that friends tend to be more similar to each other (higher homophily) in relationally mobile societies, because there are more opportunities to find like-minded friends and leave relationships if people's interests diverge (26).

There is some prior evidence that people in relationally mobile societies also share personal information more quickly (selfdisclosure) (27), give social support more frequently (8), and report higher intimacy with romantic partners (28). These "pro-active" tendencies help bind partners together, increasing the cost for either partner to pursue attractive alternatives (27). In other words, these tendencies help people retain relationships. In low relational mobility societies, relationships are stable and more difficult to change, and therefore, the task of retaining relationships is less important.

In societies with higher relational mobility, people had more trust in strangers $[r=0.36(95 \% \mathrm{BCaCI}=0.03,0.63), P=0.046]$ and higher self-esteem $[r=0.66(95 \% \mathrm{BCaCI}=0.28,0.83), P<0.001]$ (SI Appendix, Table S8). Relational mobility also explained up to $30 \%$ of between-country variance in respondents' individual-level similarity between friends and romantic partners (SI Appendix, Table S7). Self-disclosure and intimacy toward a close friend or romantic partner were also much higher in relationally mobile societies,

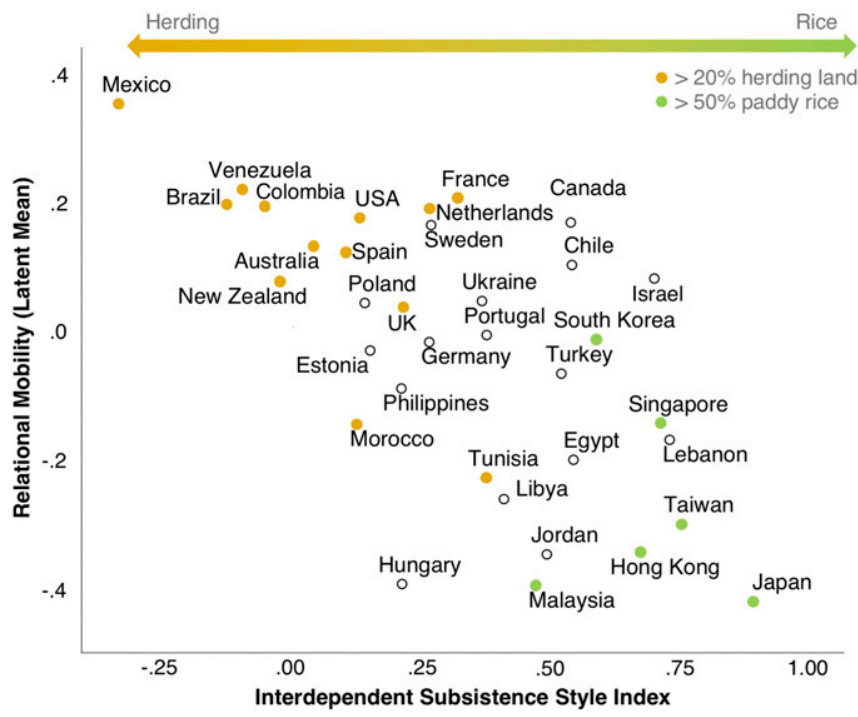

Fig. 3. Relational mobility is lower in societies that traditionally practiced more settled, interdependent subsistence styles. The index incorporates the three most widely studied subsistence styles in cross-cultural psychology: herding (relatively mobile and independent), wheat farming (more settled and interdependent), and paddy rice farming (most settled and interdependent). $S I$ Appendix, section 1.5.3 discusses how we created the index. 


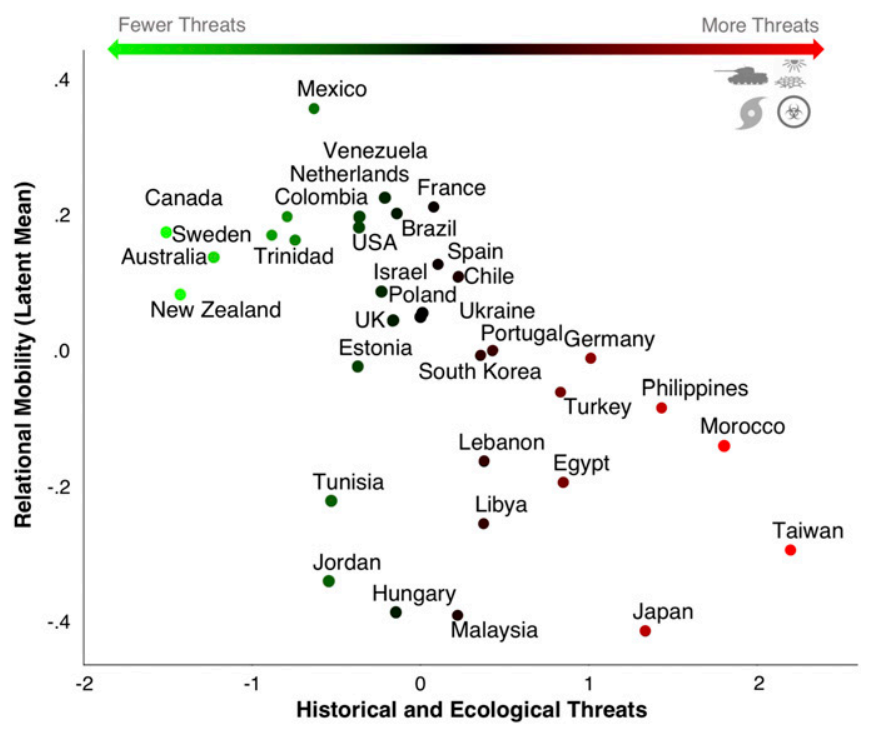

Fig. 4. Relational mobility is lower in countries that had higher historical threats, such as natural disaster, disease, greater pressure on resources, and more territorial threats. SI Appendix, section 1.4 discusses how we created the index.

with relational mobility explaining up to $54 \%$ of the variance between societies in these two dependent variables (SI Appendix, Table S9). Relationally mobile societies also reported more willingness to help out a close friend in times of personal crises (social support), explaining $23 \%$ of variance in social support between societies (SI Appendix, Table S9).

Multilevel Structural Equation Model. We brought the causes, relational mobility, and the interpersonal outcomes together in a confirmatory multilevel structural equation model (SI Appendix, Fig. S7). This model allows us to test the unique effect that each antecedent has on relational mobility as well as relational mobility's unique effect on each interpersonal outcome. In this model, we used subsistence style and threat variables adjusted for current gross domestic product per capita to test effects independent from societies' differences in wealth (SI Appendix, section 1.6). We also took into account individual-level variables, such as gender, age, and household income. The models nested societies within geographical regions, which accounts for the fact that countries are not always truly independent observations (SI Appendix, section 1.3.1).

Results for these confirmatory analyses confirmed the simple correlations presented above (SI Appendix, Table S11). Relational mobility was predicted independently by subsistence style $(\beta$ values $>-0.475, P$ values $<0.001)$ and threats $(\beta$ values $>-0.273, p$ values $<0.05)$. Among the outcomes, relational mobility predicted generalized trust, self-esteem, similarity, selfdisclosure, intimacy, and social support (SI Appendix, Table S11).

Actual Vs. Potential Relational Movement. One question that underpins this research is the distinction between actual movement and relational mobility, which measures how much choice and opportunity there are for movement between relationships in a society. We measure potential for relational movement using the relational mobility scale, which relies on peoples' perceptions of relational choice and opportunity. We reported in Convergent and Concurrent Validity of the Relational Mobility Measure that perceptions of relational mobility were correlated with actual relational movement. Therefore, why not use these measures of actual movement, which should be more objective and accurate? For example, we could measure how many new acquaintances people have made in the last month or how often people move homes (16). At least for the relationship acquisition and retention behaviors that we measure in this study, we argue that relational mobility is critical and that actual movement does not adequately measure choice and opportunity.
As an illustration, imagine that your friend is a naval officer who could be ordered to transfer to another city at any time. The knowledge that your friend may soon be transferred to another city (quite possibly against his/her own preference) will probably not increase the likelihood that you will try to work harder to retain that friend, such as by increasing intimacy or self-disclosing more. If your friend's moving away is determined by an outside force, then trying harder to retain the friendship would be useless. If, however, it is entirely up to your friend's choosing whether s/he moves away or not, then why not try to "convince" him/her to stay through expressions of intimacy or disclosing secrets? If there is choice, the relationship retention behaviors are adaptive. If there is no choice, investment is for naught.

We tested whether relational mobility is a stronger predictor of proactive relationship behaviors than measures of actual movement. To do this, we reran the multilevel model, replacing relational mobility with measures of actual movement (residential mobility and self-reported number of acquaintances met in the last month). Relational mobility more reliably predicted the outcomes in the model (SI Appendix, Table S13). Moreover, relational mobility was more reliably predicted by the theoretical antecedents. Naturally, relational mobility and actual movement between relationships will be correlated. However, this result suggests that freedom and choice in relationships are adding something beyond movement alone (SI Appendix, section 1.8 has more discussion).

Where Does Relational Mobility Sit in the Causal Chain? A critical reader might ask why we need to talk about relational mobility. The field already has concepts and measures for individualism, tightnesslooseness, and hierarchy. Does relational mobility add anything beyond these established concepts? Here, we argue that $(i)$ relational mobility is a stronger predictor of certain interpersonal outcomes and that, (ii) as a socioecological factor, relational mobility can help explain why societies have certain cultural characteristics.

First, we tested whether relational mobility predicted the interpersonal outcomes measured in this study better than previously established cultural variables. We did this by predicting self-disclosure, intimacy, and trust from relational mobility vs. cultural variables, such as individualism, cultural tightness, and cultural self-construals (SI Appendix, section 1.11.10 reports the full multilevel results). Overall, relational mobility held up well against these other variables, predicting the outcomes more consistently and strongly (SI Appendix, Table S16). This suggests that relational mobility holds unique predictive power beyond established dimensions of cultural variability.

Next, we asked whether relational mobility sits higher in the causal chain of culture or if it is more of a downstream outcome. We argue that relational mobility sits higher in the causal chain and can help explain why previous studies have found that threats $(22,29)$ and subsistence styles (18) affect culture. We reasoned that, in response to threats and different subsistence styles (distal social ecologies), humans form community structures that afford varying degrees of opportunity and freedom in relationships (i.e., relational mobility, a proximal social ecology). This variance in relational mobility then impacts the self-concepts and other cultural characteristics of people who live in those environments (SI Appendix, section 1.11).

To test this, we ran mediation models where threats and subsistence style cause relational mobility, which then causes interdependent self-construals (18) and cultural tightness-looseness $(22,29)$ (SI Appendix, section 1.11 .9 and Fig. S8). Results showed a significant indirect effect of threats and subsistence style on selfconstruals and tightness-looseness, via relational mobility. We also compared that with competing mediation models where threats and subsistence styles cause self-construals and tightness-looseness, which then cause relational mobility (SI Appendix, Fig. S9). The data did not support models where relational mobility was a downstream consequence (SI Appendix, Table S15). Overall, the model results support the theory that relational mobility is a socioecological variable, impacting how humans think and act. At the same time, we readily accept that relational mobility is not the only important feature 
of the social environment. Clearly, interdependent self-construals, norms, and other cultural settings influence behavior as well.

\section{Discussion}

Overall, the findings are consistent with the theory that relational mobility makes certain behaviors and psychological tendencies more adaptive in any given society. In particular, the findings suggest that, as relational mobility increases, it becomes more adaptive to actively invest in building interpersonal relationships.

One particularly noteworthy finding was that Latin America was high in relational mobility. Latin Americans reported behavior and psychological tendencies (such as high self-esteem) that tend to occur with relational mobility. This is noteworthy, because data suggesting that Latin America is collectivistic (30, 31) would not have predicted this. This finding might inspire researchers to delve deeper into how Latin America fits into the spectrum of human culture.

Ultimately, these results are correlational; they cannot prove that relational mobility causes these outcomes. Furthermore, reverse causality is also plausible-for example, trusting strangers could also make societies more relationally mobile. We can get more insight into cause and effect through experimental research [such as studies that manipulate relational mobility or people's perception of it (32)] and studies that track changes in the environment and mobility indicators over time (examples are in refs. 25 and 33). Agent-based simulations can also help clarify whether these behaviors are adaptive.

Future large-scale studies on relational mobility can use data that are more representative of the population in each society to test the generalizability of our findings. This applies not only to the exploratory nature of many of our analyses but also, to our sample's heavy skew toward females. Additional analysis showed that gender explains only $0.04 \%$ of the variance in relational mobility scores (SI Appendix, section 1.1.1). Individual-level age and income explained even less variance. This is plausible, because participants describe the mobility of their society, not their

1. Tönnies F (1887) Gemeinschaft und Gesellschaft (Fues's Verlag, Leipzig, Germany).

2. Kollock $P$ (1994) The emergence of exchange structures: An experimental study of uncertainty, commitment, and trust. Am J Sociol 100:313-345.

3. Yamagishi T, Yamagishi M (1994) Trust and commitment in the United States and Japan. Motiv Emot 18:129-166.

4. McElreath R (2004) Community structure, mobility, and the strength of norms in an African Society: The Sangu of Tanzania. Foundations in Human Sociality: Economic Experiments and Ethnographic Evidence from Fifteen Small-Scale Societies, eds Henrich J, et al. (Oxford Univ Press, Oxford), pp 335-355.

5. Barclay P (2016) Biological markets and the effects of partner choice on cooperation and friendship. Curr Opin Psychol 7:33-38.

6. Noë R, Hammerstein P (1994) Biological markets: Supply and demand determine the effect of partner choice in cooperation, mutualism and mating. Behav Ecol Sociobiol 35:1-11.

7. Yuki M, Schug J (2012) Relational mobility: A socio-ecological approach to personal relationships. Relationship Science: Integrating Evolutionary, Neuroscience, and Sociocultural Approaches, eds Gillath O, Adams G, Kunkel AD (Am Psychol Assoc, Washington, DC), pp 137-152.

8. Kito M, Yuki M, Thomson R (2017) Relational mobility and close relationships: A socioecological approach to explain cross-cultural differences. Pers Relatsh 24:114-130.

9. Oishi S (2014) Socioecological psychology. Annu Rev Psychol 65:581-609.

10. Yuki M, et al. (2007) Development of a Scale to Measure Perceptions of Relational Mobility in Society (Hokkaido University, Sapporo, Japan).

11. Caporael LR, Brewer MB (1991) Reviving evolutionary psychology: Biology meets society. J Soc Issues 47:187-195.

12. Brint $S$ (2001) Gemeinschaft revisited: A critique and reconstruction of the community concept. Sociol Theory 19:1-23.

13. Chiang Y-S (2010) Self-interested partner selection can lead to the emergence of fairness. Evol Hum Behav 31:265-270.

14. Adams $G$ (2005) The cultural grounding of personal relationship: Enemyship in North American and West African worlds. J Pers Soc Psychol 88:948-968.

15. Li LMW, Adams G, Kurtiş T, Hamamura T (2015) Beware of friends: The cultural psychology of relational mobility and cautious intimacy. Asian J Soc Psychol 18:124-133.

16. Oishi S, Schug J, Yuki M, Axt J (2015) The psychology of residential and relational mobilities. Handbook of Advances in Culture and Psychology, eds Gelfand MJ, Chiu C, Hong Y (Oxford Univ Press, Oxford), pp 221-272.

17. Goldschmidt W (1971) Independence as an element in pastoral social systems. Anthropol Q 44:132-142.

18. Talhelm T, et al. (2014) Large-scale psychological differences within China explained by rice versus wheat agriculture. Science 344:603-608. own mobility. However, the small samples of men make it difficult to fully test for gender differences.

This 39-society study presents a large-scale survey of relational mobility around the world. The findings suggest that subsistence styles and environmental threats can explain some of the differences across societies in relationship style. The results also suggest that free agent relationship markets encourage proactive social behaviors, like self-disclosure, intimacy, and generalized trust. We hope that future studies can continue to bring together research in biological sciences, sociology, and cross-cultural psychology to better understand how humans structure their relationships, even while people move more of their social networks into the digital world.

\section{Materials and Methods}

We measured relational mobility by recruiting 16,939 people from 39 societies to a web survey between 2014 and 2016 (Table 1); the survey was approved by the Ethics Committee of the Center for Experimental Research in Social Sciences at Hokkaido University. Recruitment was via Facebook advertisements (SI Appendix, section 1.1). Participants were directed to a landing page, where they could inform themselves about the survey and provide their consent before continuing. We sampled societies based on $(i)$ Facebook penetration rate (to maximize diversity in respondents within each country) and (ii) capturing as much variance as possible in geography and cultural blocks.

Participants rated the relational mobility of their immediate society using the relational mobility scale of Yuki et al. (10) (SI Appendix, Table S1), reported the number of new acquaintances met in the last month and how many romantic partners they have had, and completed demographic questions. They also completed measures of interpersonal intimacy (34), selfdisclosure (27), similarity (26), and social support in their relationship with either their closest friend or their romantic partner. Participants completed the survey in the majority language of their society (Table 1 and SI Appendix, section 1.2). We collected societal variables, such as gross domestic product per capita, cultural variables, romantic partner poaching frequency, and other psychological and behavioral variables, from public sources ( $S I$ Appendix, Tables S6, S8, and S10).

19. Uskul AK, Kitayama S, Nisbett RE (2008) Ecocultural basis of cognition: Farmers and fishermen are more holistic than herders. Proc Natl Acad Sci USA 105:8552-8556.

20. Van de Vliert E (2013) Climato-economic habitats support patterns of human needs, stresses, and freedoms. Behav Brain Sci 36:465-480.

21. Oishi S, Kesebir S (2012) Optimal social-networking strategy is a function of socioeconomic conditions. Psychol Sci 23:1542-1548.

22. Gelfand MJ, et al. (2011) Differences between tight and loose cultures: A 33-nation study. Science 332:1100-1104.

23. Fincher CL, Thornhill R, Murray DR, Schaller M (2008) Pathogen prevalence predicts human cross-cultural variability in individualism/collectivism. Proc Biol Sci 275:1279-1285.

24. Thomson R, Yuki M, Ito N (2015) A socio-ecological approach to national differences in online privacy concern: The role of relational mobility and trust. Comput Hum Behav 51:285-292.

25. Sato K, Yuki M (2014) The association between self-esteem and happiness differs in relationally mobile vs. stable interpersonal contexts. Front Psychol 5:1113.

26. Schug J, Yuki M, Horikawa H, Takemura K (2009) Similarity attraction and actually selecting similar others: How cross-societal differences in relational mobility affect interpersonal similarity in Japan and the USA. Asian J Soc Psychol 12:95-103.

27. Schug J, Yuki M, Maddux W (2010) Relational mobility explains between- and withinculture differences in self-disclosure to close friends. Psychol Sci 21:1471-1478.

28. Yamada J, Kito M, Yuki M (2015) Relational mobility and intimacy in friendships and romantic relationships: A cross-societal study between Canada and Japan. Jpn J Exp Soc Psychol 55:18-27.

29. Uz I (2015) The index of cultural tightness and looseness among 68 countries. J Cross Cult Psychol 46:319-335.

30. Hofstede GH (2001) Culture's Consequences: Comparing Values, Behaviors, Institutions, and Organizations Across Nations (Sage Publications, Thousand Oaks, CA).

31. Gelfand MJ, Bhawak D, Nishii L, Bechtold DJ (2004) Individualism and collectivism. Culture, Leadership and Organizations: The GLOBE Study of 62 Societies, eds House RJ, Hanges PJ, Javidan M, Dorfman PW, Gupta V (Sage, Thousand Oaks, CA), pp 473-512.

32. Oishi $S$, et al. (2007) The socioecological model of procommunity action: The benefits of residential stability. J Pers Soc Psychol 93:831-844.

33. Grossmann I, Varnum MEW (2015) Social structure, infectious diseases, disasters, secularism, and cultural change in America. Psychol Sci 26:311-324.

34. Kanemasa Y, Daibo I (2003) Three components in the triangular theory of love and intimate opposite-sex relationships. Jpn J Res Emot 10:11-24.

35. Allin1Social (2015) Facebook statistics worldwide. Allin1Social. Available at www.allin1social. com/facebook-statistics/countries/. Accessed December 18, 2015. 